\title{
Effect of sludge treatment on the bioaccumulation of nonylphenol in grass grown on sludge-amended soil
}

\author{
K. M. Nowak • V. N. Kouloumbos • \\ A. Schäffer · P. F.-X. Corvini
}

Received: 11 February 2007 / Accepted: 15 June 2007/Published online: 9 August 2007

(C) Springer-Verlag 2007

\begin{abstract}
We studied the accumulation of p353-nonylphenol residues in the biomass of grass grown in soil amended with sewage sludge submitted to various conditioning/dewatering treatments. Incubation experiments were conducted growing Poa pratensis in sludge-amended soils and applying one ${ }^{14} \mathrm{C}$-labelled isomer of nonylphenol in the different systems. More metabolites than parent compounds were recovered in both roots and leaves of the grass. The type of sludge conditioning and dewatering treatment had a slight effect on the bioaccumulation of nonylphenol and its metabolites. When the grass was cultivated in soils amended with dewatered sludge without conditioning pretreatment, an increased accumulation was observed in the roots, while the final biomass of the grass was lower.
\end{abstract}

Keywords Nonylphenol · Plant uptake ·

Bound residues - Sludge dewatering ·

Sludge conditioning $\cdot$ Sludge-amended soil

K. M. Nowak

Department of Bioremediation,

UFZ, Helmholtz Centre for Environmental Research,

Permoserstraße 15, 04318 Leipzig, Germany

V. N. Kouloumbos · A. Schäffer

Department of Biology V, Environmental Biology

and Chemodynamics, RWTH Aachen University,

Worringerweg 1, 52074 Aachen, Germany

P. F.-X. Corvini $(\square)$

Institute for Ecopreneurship, School of Life Sciences, University of Applied Sciences, Northwestern Switzerland (FHNW), Gründenstrasse 40, Muttenz 4132, Switzerland e-mail: philippe.corvini@fhnw.ch

\section{Introduction}

Among the multitude of micropollutants released into the environment, xenobiotics with estrogenic activity such as nonylphenol (degradation product of nonylphenol polyethoxylates) have focused attention (White et al. 1994; Lee and Peart 1995). Nonylphenol polyethoxylates (NnPEO) are surfactants, which are used worldwide for industry and household applications. Presently, both their production and use are subject to specific restrictions in EU, owing to the risk they represent to the environment. NPnEO are discharged via sewage water to wastewater treatment plant (WWTP), where they are degraded to a mixture of branched alkyl chain isomers of nonylphenol (Ahel et al. 1994). Through sorption processes, NP strongly accumulates in sewage sludge, which constitutes the largest by-product of wastewater treatment.

The recycling of sewage sludge for amendment of arable soil is a low-cost common practice possessing many beneficial effects. Sludge contains high amount of elemental nutrients (e.g., nitrogen, phosphorus, and potassium) and organic matter, which can improve the physico-chemical properties of soil and thus, enhance the crop growth. Nevertheless, the presence of xenobiotics and heavy metals in sludge to be used as fertilizer poses safety problem. The concentration of NP in stabilized sludges varies considerably and is strongly dependent on sludge treatment processes used. For instance, in Spain the amounts of NP fluctuate between $50 \mathrm{mg} \mathrm{kg}^{-1}$ in $\mathrm{dw}$ of sludges without digestion and $2,800 \mathrm{mg} \mathrm{kg}^{-1} \mathrm{dw}$ for sludges after aerobic digestion (Gomez-Rico et al. 2007). Due to the establishment of limitations in the use of NPnEO as surfactants in several countries, the concentration of NP in sludges is gradually decreasing. Solid handling processes such as conditioning and dewatering are commonly applied for the removal of 
water from the sludges, usually after thickening and stabilization. Among the various conditioning processes, chemical conditioning by either organic or inorganic chemicals is often applied in Germany and other EU countries. Physical conditioning by means of freeze-thawing is rather restricted to cooler climatic zones, where it is economically feasible. It was reported that the conditioning of sludge before dewatering influences the amount of contaminants remaining sorbed to the sludge and it may impact the fate of contaminant in sludge amended soil (Ivashechkin et al. 2004). For instance, the liming of sludge leads to desorption of alkylphenols from sludge matrix through the deprotonation of the phenolic group. The soil amendment with sludge containing xenobiotics, can also lead to the propagation of the pollution to other compartments of the environment such as groundwater and plant. The fate of these compounds in sludgeamended soil-plants system depends on many factors such as the physico-chemical properties of sludge and soil and the type of plants. For instance, the growth of plants affects the biodegradation of organic compounds such as polycyclic aromatic hydrocarbons (Ferro et al. 1994). Plant roots exudates also enhance microbial activity, which plays a key role in degradation of xenobiotics (Davis et al. 2002). Consecutively to their uptake, pollutants can be degraded by plant enzymes or immobilized in vacuoles and cell walls as "bound residues" (Burken 2003; Pilon-Smits 2005). In the case of NP, relatively few studies on its uptake by plants were carried out and they are limited to experiments with suspended cells or hydroponic cultures. The toxicity, uptake and metabolism of linear alkyl chain NP was investigated in cell suspension cultures of different plants (Bokern et al. 1998). In crested wheatgrass (Agropyron cristatum) cultivated in hydroponic system, most of NP residues were recovered in the roots (Doucette et al. 2005). Detailed studies concerning the bioaccumulation of NP in plant-soil systems are still lacking.

The present study relates the accumulation of NP and its residues in the different biomass fractions of the grass $\mathrm{Poa}$ pratensis grown on sludge-amended soils over a period of 82 days. A particular attention is paid to the effects of various sludge conditioning/dewatering treatments (freezethawing, centrifugation, and polymer addition) on the further fate of NP in P. pratensis grown on sludge-amended soil systems.

\section{Experimental}

Preparation and incubation of plants/sludge-amended soil systems

Liquid sludge was sampled from local wastewater treatment plants (Aachen-Soers) after the thickening and stabilization steps. These sludge samples were spiked with a radiolabeled single isomer of NP, i.e. the 4-[1-ethyl-1,3dimethylpentyl]phenol (p353NP), which was synthesized via a Friedel-Crafts alkylation according to the method of Vinken et al. (2002). A single isomer of NP was used to circumvent analytical problems related to the technical mixture of NP (tNP). In order to prevent analytical problems related to the high hydrophobicity of NP, the study was carried out with a radiolabeled form of p353NP enabling the precise tracing of the compound. The spiked amount of p353NP was adjusted to approximately $15 \mathrm{mg}$ $\mathrm{NP} \mathrm{kg}{ }^{-1}$ in the final sludge/soil mixture, corresponding to a total of $166.67 \mathrm{kBq}$ per assay. Spiking of NP was performed by adding $150 \mu \mathrm{L}$ of NP dissolved in ethanol to the liquid sludges and the mixture was immediately stirred. After spiking, liquid sludges were directly conditioned and dewatered at lab-scale. For this study, two sludges were conditioned chemically (polymer addition) and physically (freeze-thawing) prior to the dewatering step. The cationic polymer (Nerolan Wassertechnik GmbH Krefeld, Germany) was added to the sludge at a concentration of $8.5 \mathrm{~g} \mathrm{~kg}^{-1}$ dry matter of sludge for chemical conditioning, while the physical conditioning was carried out by freezing the liquid sludges overnight at $-21^{\circ} \mathrm{C}$ in a freezer and successive thawing. After these steps sludge samples were dewatered by means of a centrifugation step $(16,000 \mathrm{~g}$, for $60 \mathrm{~min}$ ). The two conditioned and dewatered sludges, and one sludge, which was only dewatered, were mixed separately with $13 \mathrm{~g}$ of sandy loam soil and the seeds of Poa pratensis (30 seeds per assay) were added to the mixture. Incubation for 82 days under continuous aeration and thermostated conditions $\left(20^{\circ} \mathrm{C}\right)$ was carried out in flowthrough systems in triplicate series (Telscher et al. 2005). High Pressure Sodium (HPS) lamps were used as light source ( $8 \mathrm{~h} \mathrm{light/day).} \mathrm{During} \mathrm{the} \mathrm{incubation,} \mathrm{the} \mathrm{miner-}$ alization rates of ${ }^{14} \mathrm{C}$-labelled $\mathrm{NP}$ were determined by trapping the ${ }^{14} \mathrm{CO}_{2}$ in traps containing $2 \mathrm{M} \mathrm{NaOH}$. Aliquots of $1 \mathrm{ml}$ from the $\mathrm{NaOH}$ solution were sampled weekly for radioactivity measurement using Liquid Scintillation Counting (LSC, see "Analytical procedures").

\section{Incubated systems analyses (soil and plant analyses)}

Residues extraction from soil

The working out of soil samples consisted of liquid-liquid sequential extraction using acetone $(25 \mathrm{~mL}), n$-hexane $(25 \mathrm{~mL})$, methanol $(25 \mathrm{~mL})$, twice $\mathrm{NaOH}(0.1 \mathrm{M}, 25 \mathrm{~mL})$, and methanol $(25 \mathrm{~mL})$. During the soil extraction, the radioactivity of the extracts was measured between each extraction step using LSC before pooling the organic extracts and alkali solutions separately. Non-extractable 
residues remaining in soil (bound to humins and minerals) after sequential extraction procedure were kept for the analysis of radioactivity contained in solid matrix (see "Analytical procedures").

\section{Residues extraction from plants}

At the end of incubation period the leaves of grass were separated from roots and were ground separately using a homogenizer. The roots of grass were cleaned up with water in order to remove soil particles. The leaves and roots of grass were extracted separately thrice using a solvent system consisting of dichloromethane/methanol/water (1:2:0.8). The extracts were decanted into two phases (methanol/water and dichloromethane phases) by adding $265 \mu \mathrm{L}$ of water and 265 $\mu \mathrm{L}$ of dichloromethane per mililiter of extract. The two phases were concentrated separately and analyzed by means of HPLC (see section Analytical procedures). After liquidliquid extraction, grass containing non-extractible residues of NP was kept to determine the amount of bound residues as for soil samples.

\section{Analytical procedures}

\section{Liquid scintillation counting}

Unless stated otherwise, aliquots of different fractions were added to $4 \mathrm{~mL}$ of a Lumasafe scintillation cocktail (Lumac*LSC BV, Netherlands) for LSC. Vials were analyzed by means of a Beckman LS 5000TD liquid scintillation counter.

Analysis of radioactivity contained in solid matrix samples

Aliquots of approximately $100 \mathrm{mg}$ solids were packed in cellulose and combusted in a biological oxidizer OX500 (RJ Harvey Instrument Corporation, USA). Resulting ${ }^{14} \mathrm{CO}_{2}$ was trapped in Carbomax Plus LSC cocktail (Lumac*LSC BV, Netherlands), and was subjected to LSC.

High performance liquid chromatography (HPLC)

The extracts from soil and grass samples were analyzed using a HPLC Series 1100 system (Hewlett Packard) equipped with an auto-injector, a degasser, a diode array detector, and an online liquid scintillation radio flow detector (Ramona Star; Raytest, Straubenhardt, Germany) with a cell volume of $1,300 \mu \mathrm{L}$. The flow rate of the scintillation cocktail (Quicksafe Flow 2; Zinsser Analytic $\mathrm{GmbH}$, Frankfurt, Germany) for the radio detector was $2 \mathrm{~mL} \mathrm{~min}^{-1}$. Chromatographic separation was performed on a Nucleosil C18 column $(250 \times 4 \mathrm{~mm})(\mathrm{CS}$ Chromatographie Service $\mathrm{GmbH}$, Germany) at $35^{\circ} \mathrm{C}$ using a flow of $1 \mathrm{~mL} \mathrm{~min}{ }^{-1}$. Mobile phase consisted of water (A) and acetonitrile (B). Samples were separated using a gradient program as follows: $25 \% \mathrm{~B}$ in $\mathrm{A}$ with linear gradient to $90 \%$ B during $22 \mathrm{~min}$, after $90 \%$ B isocratic for $5 \mathrm{~min}$. Finally, the system returned to its initial conditions (25\% B in A) within $10 \mathrm{~min}$, and was kept in this composition for 3 min before the next run started.

\section{Results and discussion}

Distribution of ${ }^{14} \mathrm{C}$ in the various fraction of soil and in grass

The distribution of radioactivity recovered from the sludgeamended soil-grass systems at the end of incubation is presented in Fig. 1. The sludge treatment did not drastically affect the general fate of NP. Only slight differences were observed. The highest amount of ${ }^{14} \mathrm{C}$-labelled extractible compounds was detected in organic extracts obtained from soil amended with dewatered sludge without pre conditioning. At the opposite, soil amended with physically (freeze thawing) and chemically conditioned (polymer addition) sludge contained higher amount of $\mathrm{NaOH}$ extractible and bound residues, correspondingly. Mineralization and uptake by plants were low and remained below 3 and $1.7 \%$, respectively, while the amount of bound residues in amended soils accounted for between 20 and $40 \%$ of the initially applied radioactivity. The amounts of mineralized ${ }^{14} \mathrm{C}$ were similar for the different sludge treatments. A slightly lower total uptake by Poa pratensis was observed in the systems amended with simply dewatered sludge (1.0\% in comparison with 1.2 and

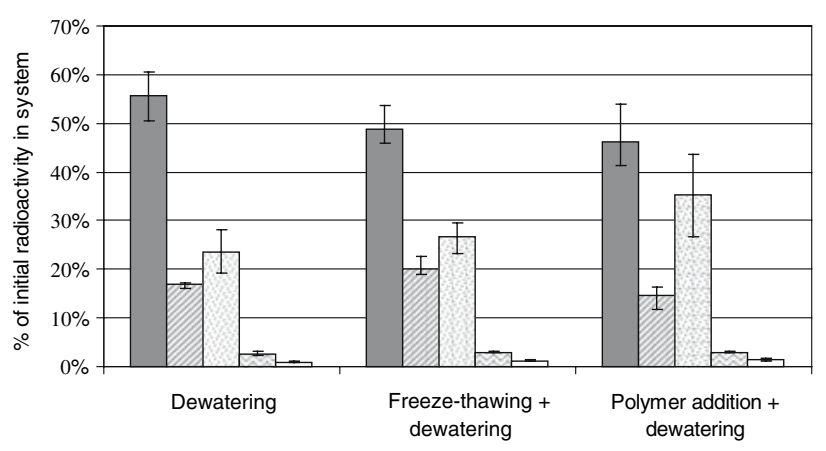

Fig. 1 Distribution of radioactivity between the various fractions of soil and in the whole plants in systems amended with differently treated sludges (dewatering, freeze-thawing and dewatering, and polymer addition and dewatering). Radioactivity recovered in: organic extract (filled bars); $\mathrm{NaOH}$ extract, (dashed bars); bound residues in soil (dotted bars); mineralized fraction (wavy bars); Plant (open bars). Error bars represent the minima and maxima 
$1.4 \%$ in systems amended with freeze-thawed and polymer conditioned sludge correspondingly).

\section{Biomass of Poa pratensis}

Dry leaves and roots of Poa pratensis were weighed separately. The biomass of leaves of grass in each treatment was higher than roots (Fig. 2). Both the biomass of roots and leaves of grass grown on soil amended with solely dewatered sludge was the lowest in comparison with the biomass $P$. pratensis in two conditioned sludge systems (significantly lower only in comparison with polymer conditioned sludge systems), although the same amounts of sludge dry weight was applied in all the systems. The highest average biomass of grass was observed in the systems amended with sludge conditioned by polymer addition. No differences in roots biomass were observed between the systems amended with conditioned sludge. The lowest biomass of leaves and roots, in system where sludge was not conditioned-may be connected with the high amounts of extractible NP residues found in the extracts from the corresponding soil.

This would be indicative for toxic effects on the plants resulting from the high bioavailable fraction of NP residues in these systems. According to Bokern et al. (1998), high concentration of NP can be toxic for plants. By investigating the toxicity, uptake and metabolism of $4-n$-NP in root cultures Lupinus hartwegii, it was demonstrated that $\mathrm{NP}$ is toxic at $0.1 \mathrm{mM}\left(22 \mathrm{mg} \mathrm{kg}^{-1}\right)$, resulting in $50 \%$ growth reduction. The distinction between parent compounds and degradation products could not be achieved in a reliable way using HPLC analyses in the present study. Some NP degradation occurred during the storage of these extracts from soil and did not allow the exact quantification of the amount of remaining NP in extracts from soil/sludge.

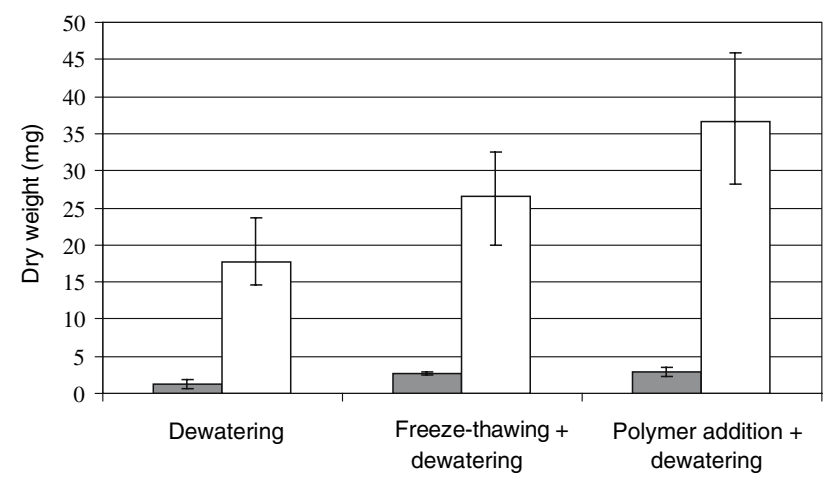

Fig. 2 Biomass of leaves and roots of grass at the end of incubation in presence of three differently treated sludges (dewatering, freezethawing and dewatering, polymer addition and dewatering). Dry weight: roots (filled bars) and leaves (open bars). Error bars represent the minima and maxima
Concentration of total radioactivity (NP and its metabolites) in biomass of Poa pratensis

As mentioned in the experimental section, the combined organic extract of grass after the extraction steps was decanted into two phases and the total radioactivity (NP and metabolites) was measured in each phase separately using LSC. After the extraction procedures, the biomass was combusted in order to determine the bound residues. The sum of radioactivity uptake separately in roots and leaves is presented in Fig. 3 as uptake per biomass.

Independent of the sludge treatment, radioactivity was above three times more accumulated in roots than in leaves of the grass. The amounts of total radioactivity uptaken by leaves and roots of grass were not significantly different in tested sludge treatment. The trend for higher ${ }^{14} \mathrm{C}$ concentration, both in leaves and roots of Poa pratensis grown in presence of dewatered sludge without pre-conditioning was observed. This can be related with the higher amount of extractible residues in this case. Furthermore, this tends to corroborate the toxicity hypothesis formulated to interpret the lower amounts of biomass obtained for these series.

NP in dichloromethane extracts of grass

The fraction of the total accumulated radioactivity in grass that was extracted by using relatively apolar solvent, i.e. dichloromethane, is shown in Fig. 4.

The analysis in deeper details of these dichloromethane extracts was carried out using HPLC coupled to radiodetection (Fig. 4b). As exemplarily shown for an extract prepared from leaves of Poa pratensis grown on soil amended with non-conditioned sludge, radioactivity recovered in all these extracts from leaves or roots consisted mainly of nonylphenol (RT $22 \mathrm{~min}$ ) independent of the sludge treatment. The small peaks eluting after $25 \mathrm{~min}$

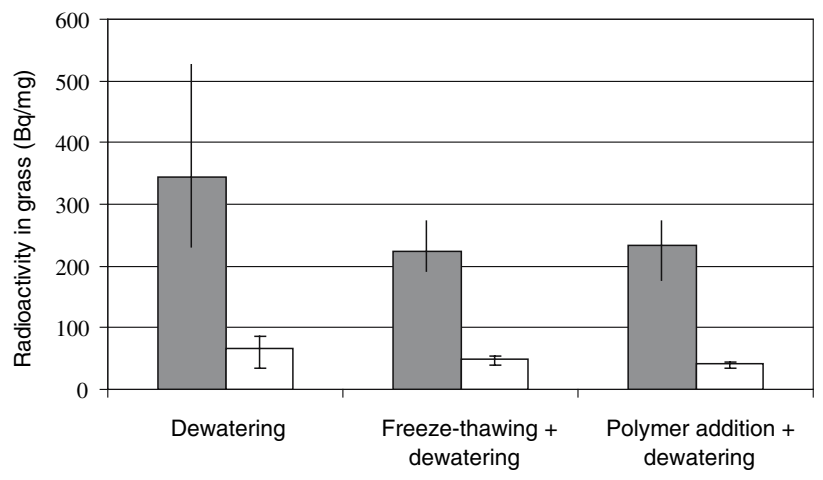

Fig. 3 Radioactivity distribution in the total biomass of Poa pratensis grown in soils amended with three differently treated sludges. Radioactivity recovered in roots (filled bars); and in leaves (open bars) 
was tentatively attributed to the dialkylated form of NP, which is a by-product of NP synthesis and elutes at this RT under similar chromatographic conditions. On the whole, the concentration of NP in roots was much higher than in leaves of Poa pratensis in each treatment (data not shown) and these observations are in agreement with those of studies demonstrating the higher accumulation of linear NP in roots than in shoot of two tested species of cell cultures (Lycopersicon esculentum and Artiplex hortensis) (Bokern et al. 1998). In all series, NP accounted for the $5-15 \%$ of the accumulated radioactivity, both in roots and leaves, and without any significant difference according to the sludge treatment.

NP metabolites in methanol-water extracts of grass

As for the "apolar" phase, the amount of radioactivity recovered in methanol-water phase of extracts from the roots per biomass was higher than in the leaves of Poa pratensis (not shown). The fraction of the total accumulated radioactivity in grass that was extracted by methanol-water is shown in Fig. 5. The trend for the higher proportion of methanol-water extractible residues was observed in the leaves and roots of grass grown on soil amended with nonconditioned sludge. Ascertainment of the compounds present in the extracts by means of HPLC-radiodetection
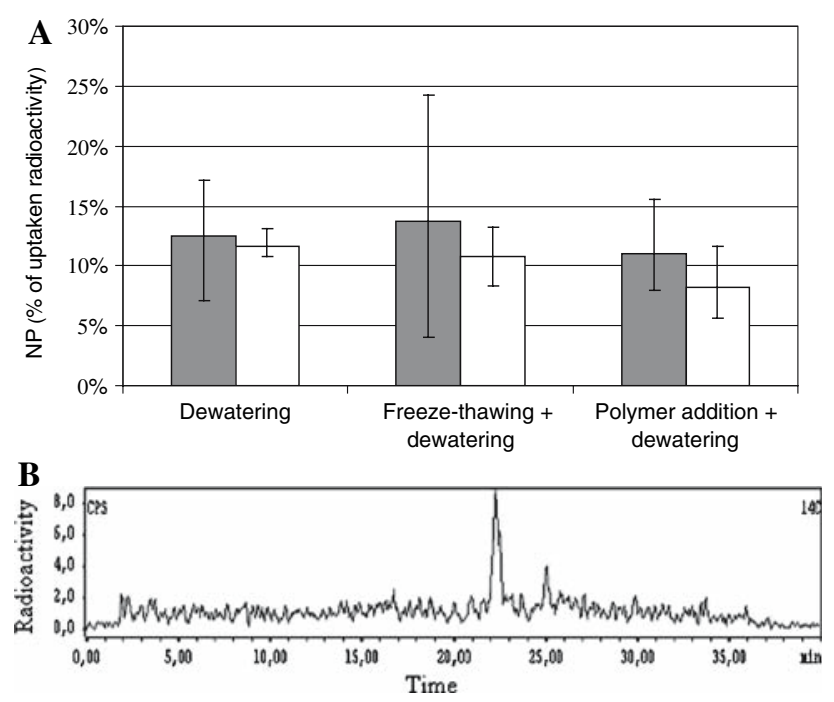

Fig. $4 \mathrm{CH}_{2} \mathrm{Cl}_{2}$ extractible residues of $\mathrm{NP}$ in roots and leaves: radioactivity amounts, parent compound and metabolites. 4A: Concentration of NP in dichloromethane extracts of grass grown on systems amended with three differently conditioned/dewatered sludges. Radioactivity measured by means of LSC in roots (filled bars) and leaves (open bars) of Poa pratensis. 4B: Typical HPLC radiochromatogram of dichloromethane extract from leaves or roots of Poa pratensis. The chromatogram corresponds to the extract obtained from leaves of grass grown in presence of dewatered sludge. Radioactivity is expressed in counts per second (CPS) showed that the total radioactivity measured by means LSC consisted exclusively of degradation products of NP, independently of the sludge treatment applied (Fig. 5b). For both extracts prepared from leaves and roots, the highest peak was eluting between 2 and $12 \mathrm{~min}$ and corresponded to a mixture of undefined polar metabolites of NP.

Non-extractible residues of ${ }^{14} \mathrm{C}$ in leaves and roots of grass

After extraction procedures, the plant samples were combusted using oxidizer in order to determine the amount of non-extractible radioactive compounds. The amount of bound residues as part of the total radioactivity taken up by the biomass is presented in Fig. 6. A significant amount of residues not extractible using dichloromethane or methanol-water was accumulated in the leaves and roots of grass for all the systems. In leaves, the radioactivity corresponding to bound residues was predominant in systems containing conditioned sludge, while it was generally lower for those containing solely dewatered sludge. As it can be seen in Fig. 4, 5, and 6, no significant differences in the type of residues (NP, metabolized NP or bound residues) were found in the roots of grass grown on soils amended with differently treated sludges.
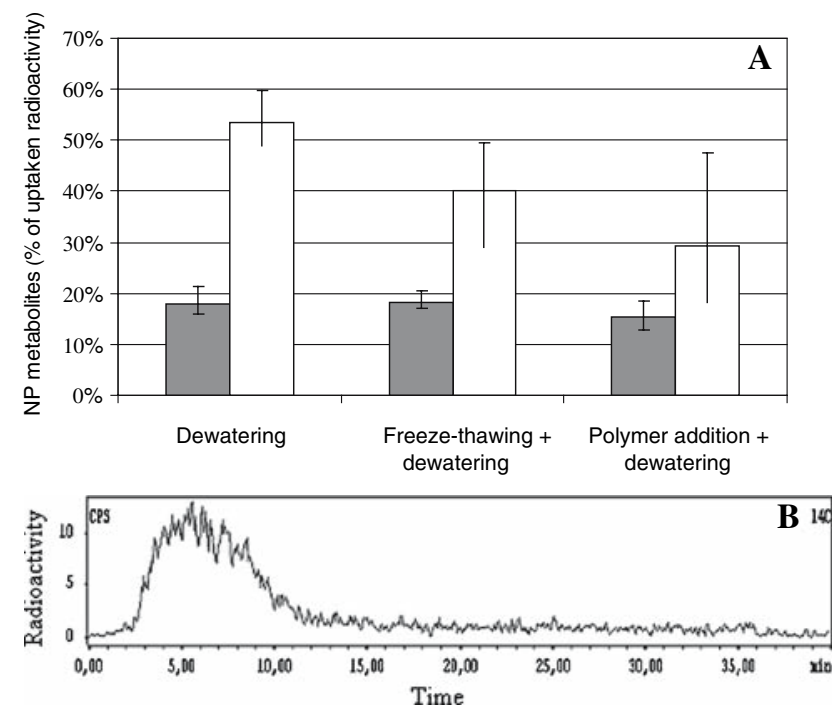

Fig. 5 Methanol-water extractible residues of NP in roots and leaves: radioactivity amounts, parent compound and metabolites. 5A: Concentration of NP in methanol-water extracts of grass grown on systems amended with three differently conditioned/dewatered sludges. Radioactivity measured by means of LSC in roots (filled bars) and leaves (open bars) of Poa pratensis. 5B: Typical HPLC radiochromatogram of methanol-water extract from leaves or roots of Poa pratensis. The chromatogram corresponds to the extract obtained from leaves of grass grown in presence of dewatered sludge. Radioactivity is expressed in counts per second (CPS) 


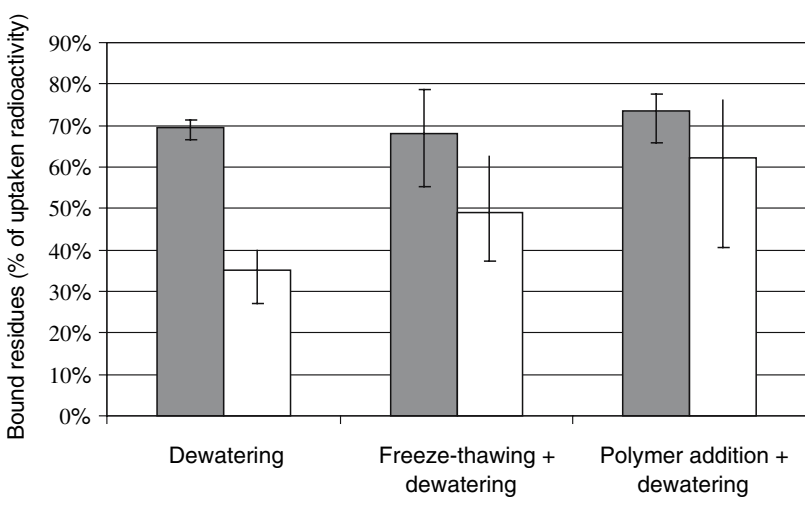

Fig. 6 Non-extractible residues of ${ }^{14} \mathrm{C}-\mathrm{NP}$ in leaves and roots of grass grown in soils amended with three differently treated sludges. Radioactivity associated to the biomass roots (filled bars) and leaves (open bars) of Poa pratensis after extractions by means of dichloromethane and methanol-water

\section{Conclusion}

In the present study, we demonstrated that the uptake of NP by Poa pratensis is very low (less than $650 \mu \mathrm{g} \mathrm{g}^{-1}$ dry weight). NP is very hydrophobic compound $\left(\log K_{\mathrm{ow}}=\right.$ 3.8 - 4.8 according to Vazquez-Duhalt et al. 2005), which displays low mobility and bioavailability in soil. Interestingly, these results point out to the potential importance of sludge conditioning/dewatering treatment on the further fate of contaminants in plant/sludge-amended soil. The highest amount of residues extractible in organic solvent was observed in soil containing sludge, which was not conditioned prior to the dewatering step. In this case, the accumulation of radioactive residues per biomass in the respective roots was high and the biomass of the grass in these systems was low, possibly due to an effect related to the relatively high availability of NP. We also demonstrated that the concentration of metabolites and bound residues in the grass was much higher than parent compounds and was indicative for metabolism in plant tissues. Extractible metabolites of NP was the predominant form of accumulated residues in the leaves of grass with low biomass (on soil amended with solely dewatered sludge), while the bound residues predominated in the leaves tissues when polymer or freeze-thaw conditioning had been applied to the amended sludge. To the best of our knowledge, this is the first report on the influence of sludge conditioning/dewatering on the further uptake of micropollutants by plants grown in sludge-amended soils. In order to elucidate the mechanisms underlying our observations, detailed studies on the fate of NP in soils amended with different conditioned/dewatered sludges are necessary.

Acknowledgments We thank the European Commission for funding AQUAbase under the Human Resources and Mobility Activity within the 6th Framework Programme under contract number MESTCT-2004-505169. The scientific advices of Dr. B Schmidt are also gratefully acknowledged.

\section{References}

Ahel M, Giger W, Koch M (1994) Behaviour of alkylphenol polyethoxylate surfactants in the aquatic environment I. Occurrence and transformation in sewage treatment. Water Res 28:1131-1142

Bokern M, Raid P, Harms H (1998) Toxicity, uptake and metabolism of 4- $n$-NP in root cultures and intact plants under septic and aseptic conditions. Environ Sci Pollut Res Int 5:21-27

Burken JG (2003) Uptake and metabolism of organic compounds: green liver model. In: SC McCutcheon JL Schnoor (eds) Phytoremediation: transformation and control of contaminants. Wiley, New York, pp 59-84

Davis CD, Castro-Diaz S, Zhang Q, Erickson LE (2002) Benefits of vegetation for soils with organic contaminants. Crit Rev Plant Sci 21:457-491

Doucette WJ, Wheeler BR, Chard JK, Bugbee B, Naylor CG, Carbone JP, Sims RC (2005) Uptake of nonylphenol and nonylphenol ethoxylates by crested wheatgrass. Environ Toxicol Chem 11:2965-2972

Ferro AM, Sims RC, Bugbee B (1994) Hycrest crested wheatgrass accelerates the degradation of pentachlorophenol in soil. $\mathrm{J}$ Environ Qual 23:272-279

Gomez-Rico MF, Font R, Aracil I, Fullana A (2007) Analysis of organic pollutants in sewage sludges from the Valencian community (Spain). Arch Environ Contam Toxicol 52:306-316

Ivashechkin P, Corvini PFX, Dohmann M (2004) Behaviour of endocrine disrupting chemicals during the treatment of municipal sewage sludge. Water Sci Technol 50:141-147

Lee HB, Peart TE (1995) Determination of 4-nonylphenol in effluent and sewage sludge from sewage treatment plants. Anal Chem 67:1976-1980

Pilon-Smits E (2005) Phytoremediation. Annu Rev Plant Biol 56:1539

Telscher MJH, Schuller U, Schmidt B, Schäffer A (2005) Occurence of a nitro metabolite of a defined nonylphenol isomer in soil/ sewage sludge mixtures. Environ Sci Technol 39:7896-7900

Vazquez-Duhalt R, Marquez-Rocha F, Ponce E, Licea AF, Viana MT (2005) Nonylphenol, an integrated vision of a pollutant. Scientific review. Appl Ecol Environ Res 4:1-25

Vinken R, Schmidt B, Schäffer A (2002) Synthesis of tertiary ${ }^{14} \mathrm{C}$ labelled nonylphenol isomers. J Label Compd Radiopharm 45:1253-1263

White R, Jobling S, Hoare SA, Sumpter JP, Parker MG (1994) Environmentally persistent alkylphenol compounds are estrogenic. Endocrinology 135:175-182 